\title{
Médiatisation d'une activité déconnectée : enseignements tirés d'une re-scénarisation à la volée et dans l'urgence
}

\section{Mediatization of a disconnected activity: lessons learned from re-scripting on the fly and in an emergency}

\section{Mediatización de una actividad desconectada: lecciones aprendidas de la reescritura sobre la marcha y en una emergencia}

Nicolas Szilas, Ph. D., maître d'enseignement et de recherche TECFA-FPSE, Université de Genève, Suisse

nicolas.szilas@unige.ch

Kim Le, M. Sc.

TECFA-FPSE, Université de Genève, Suisse

thi.le@unige.ch

\section{RÉSUMÉ}

Cet article expose la transposition en modalité à distance d'une activité de prototypage papier pour la conception de jeux vidéo se déroulant initialement en présentiel dans le cadre d'un cursus hybride. Le passage dans l'urgence au format numérique de cette activité nous a amenés à médiatiser la collaboration tangible et présentielle vers la modalité distancielle, sans possibilité de re-conception globale du scénario pédagogique. Nous décrivons le dispositif mis en place, le déroulement de l'activité, puis analysons ses succès et limites à partir de nos analyses et des retours des apprenants.

Mots-clés : prototype papier, jeux vidéo, formation hybride, médiatisation, scénarisation pédagogique 
This article describes the transposition of a paper prototyping activity for the design of video games initially taking place in a face-to-face mode into a remote setting as part of a blended curriculum. The emergency switch to digital mode led us to mediatize face-to-face tangible communication to a distance mode, without any possibility for a global redesign of the educational scenario.

Keywords: paper prototype, video game, blended learning, mediatization, instructional design

RESUMEN

Este artículo describe la transposición a modo remoto de una actividad prevista inicialmente en formato presencial (la creación de prototipos en papel para el diseño de videojuegos), como parte de un plan de estudios híbrido. El cambio en un contexto de emergencia al modo digital nos llevó a adoptar un enfoque de mediatización de esta actividad, una adaptación directa de la comunicación cara a cara a la comunicación a distancia, sin la posibilidad de una nueva concepción global del escenario educativo.

Palabras clave: prototipo en papel, videojuego, formación híbrida, mediatización, diseño pedagógico

\section{Contexte}

Les formations dites hybrides consistent à mêler dans un même cours à la fois des séances en présentiel et des périodes à distance (Charlier, Deschryver et Peraya, 2006). Le mélange peut fortement varier d'un scénario pédagogique à l'autre, mais l'idée générale reste la même: combiner le meilleur des deux mondes, typiquement la flexibilité de la distance avec l'engagement social et émotionnel des activités en présence (Conrad, 2005). C'est dans ce contexte que se situe l'activité que nous souhaitons décrire ici, à savoir une formation dans laquelle tous les cours se déroulent en mode hybride. II s'agit du master MALTT de l'Université de Genève, dans lequel six périodes se succèdent, chacune comprenant une semaine en présentiel et quatre semaines à distance. Ce cursus comprend un cours sur les jeux vidéo pédagogiques, qui vise notamment à amener les étudiants à pratiquer une démarche de conception selon une pédagogie par projet. Dans la dernière période de cours, une semaine intensive en présence est prévue pour la création en groupe de prototypes papier des jeux vidéo, qui seront ensuite développés pendant les quatre semaines à distance'. Or, pour l'année 2020, étant donnée la crise sanitaire liée à la COVID-19, nous avons dû transposer cette semaine présentielle de prototypage papier en atelier à distance. C'est de cette transition abrupte que nous souhaiterions parler dans cet article, de ses difficultés, de ses paradoxes, mais aussi de ses opportunités.

\section{Re-scénariser à la volée}

L'obligation de passer en distanciel sans avoir pu l'anticiper va à l'encontre d'une scénarisation pédagogique de qualité. En effet, bien que ce cours aurait pu être totalement à distance en adoptant dès

\footnotetext{
${ }^{1}$ On trouvera les meilleures réalisations des étudiants à cette adresse : https://tecfa.unige.ch/jeux/jeux/\#617+category:cours-vip).
} 
le départ un scénario pédagogique adéquat, le scénario pédagogique original n'est pas a priori adapté à un changement de modalité en cours de route (à la volée). En l'occurrence, nous étions déjà dans une formation hybride et l'activité prévue en présence avait été choisie précisément par rapport à cette modalité présentielle. Les concepteurs du cours considéraient que l'activité exploitait au maximum les bénéfices de cette présence. Détaillons maintenant cette activité et ses caractéristiques.

Le prototypage papier est une activité de conception aujourd'hui reconnue dans le processus global de création d'un jeu vidéo (Fullerton, 2008; Schell, 2008). L'activité consiste à se réunir en groupe de projet et à réaliser un espace de jeu uniquement à partir de matériel tangible : papier, crayon, carton, pions, etc. en les assemblant de diverses manières (colle, ruban adhésif, etc.) afin de créer une maquette très basique du jeu visé. Puis, cette maquette est utilisée pour tester le jeu avec une personne qui joue le rôle du joueur (s'il n'y a qu'un joueur) et les autres participants manipulent le prototype pour simuler les règles du jeu. II y a donc deux phases dans un prototypage papier : la création et le test. La caractéristique première d'un prototype papier est sa rapidité : rapidité de conception, rapidité de modification. L'aspect tangible proprement dit n'est pas nécessairement mis en avant par les game designers, mais le fait de pouvoir manipuler directement les éléments du jeu est corrélé à un sentiment de plaisir indéniable, car il permet, pour une fois, de sortir du cadre de l'écran informatique, de s'en libérer pour une activité plus concrète, voire régressive (retour aux jeux de l'enfant, même si les jeux de plateau sont aujourd'hui très populaires). Enfin, la collaboration est essentielle dans cette activité. Elle intervient à l'intérieur du groupe pour discuter des choix de conception, cette discussion s'appuyant directement sur le jeu : un concepteur ne va pas se contenter d'expliquer comment il voit telle règle dans le futur jeu, il va la mettre en scène directement en manipulant ses éléments en construction. La collaboration intervient aussi entre les groupes : dans le scénario pédagogique original, chaque première exécution entière du jeu est " publique » (toute la classe y assiste) et le joueur est choisi hors du groupe de conception. Dans les itérations suivantes, le joueur sélectionné est aussi hors du groupe, typiquement l'enseignant ou l'assistant.

Puisque la décision de fermer l'université a été prise une semaine avant le démarrage de la séance présentielle, les marges de manœuvre étaient faibles. Sinon totalement abandonner l'idée d'utiliser la semaine intensive pour avancer en collaboration le travail de conception, la seule option possible était donc la « transposition du présentiel au distanciel » (Villiot-Leclercq, 2020), sans pouvoir réellement mettre en œuvre une ingénierie pédagogique globale. Ce faisant, non seulement, comme expliqué ci-dessus, cette transformation à la volée allait contre le principe d'une scénarisation bien pensée, mais elle allait même, ironiquement, contre notre scénarisation : le prototypage papier est, par excellence, une activité présentielle par ses caractéristiques collaboratives, tangibles et immédiates qui viennent d'être explicitées.

La mission était donc celle d'une médiatisation (Peraya, 2008) de l'activité de prototypage papier : il s'agissait de transposer un dispositif articulé autour du prototypage papier vers un autre dispositif à distance utilisant les technologies en ligne. Le défi s'avérait grand, car autant on dispose aujourd'hui de connaissances importantes concernant la médiatisation de contenus multimédias ou de processus de communication, autant la médiatisation d'activités de manipulation est plus rare, mais on peut penser, en physique, aux laboratoires virtuels et aux expérimentations à distance (remote experiments) (Scanlon, Colwell, Cooper et Di Paolo, 2004). 


\section{Recherche d'outils pour (re)construire un dispositif efficace}

La question des logiciels ou services en ligne s'est posée très rapidement. Tout d'abord, il nous est apparu qu'il n'existait pas de plateforme " clefs en main » dédiée à la manipulation collaborative d'objets réels ou virtuels. Nous précisons « clefs en main », car il est probable que des projets de recherche en téléprésence aient pu proposer des dispositifs approchants, mais il nous fallait une solution immédiatement opérationnelle.

Dès lors, il a fallu explorer des outils approchants qu'il nous faudrait au besoin détourner de leur usage premier pour les adapter à notre cas particulier. Selon la dimension du prototypage papier que l'on considère, on peut se diriger vers telle ou telle famille d'outils. Par exemple, si c'est la dimension collaborative que l'on privilégie, alors on pourra regarder du côté des outils de visioconférence, que la crise sanitaire de 2020 a largement popularisés. Mais que faire alors de l'activité de manipulation? On pourrait la conserver physiquement chez l'un des participants qui, du même coup, effectuerait toutes les manipulations pour le groupe. Si cette solution est séduisante, car elle garde intacte la matérialité du prototype papier, elle rompt la symétrie des rôles lors de la situation collaborative initiale, conférant un rôle particulier à l'un des acteurs. De plus, on pouvait craindre que, pour les autres acteurs, la manipulation indirecte du prototype puisse être gênante, voire bloquante. Enfin, du point de vue technique, il aurait fallu assurer une qualité de filmage du prototype, avec certainement la nécessité de fixer à un pied la caméra, une configuration loin d'être évidente à mettre en place chez les apprenants. Dans l'optique de rétablir la symétrie, la possibilité de reproduire le prototype papier chez chacun des participants est envisageable en théorie, mais en pratique, répercuter chaque modification chez tous les participants n'est guère compatible avec la réactivité recherchée avec ce type d'approche de conception.

Ainsi n'avons-nous pas retenu cette solution. En effet, nous avons souhaité considérer avant tout la dimension manipulatrice du prototypage papier, et donc virtualiser le prototype ${ }^{2}$. Plusieurs solutions se présentent alors, la première étant finalement un «retour aux sources », puisqu'en virtualisant un prototype, qui est lui-même une matérialisation d'un (futur) objet numérique, on peut penser qu'on revient à la case départ et qu'on réalise un prototype informatique. Ainsi, il existe des outils de prototypage virtuel destiné à la construction de sites Web (Axure, n.d.; Figma, n.d.; Marvel, n.d.). Une comparaison entre prototypage réel et prototypage virtuel montre que ces outils, sans surpasser le prototypage papier sur tous les plans, ont des qualités indéniables en ce qui concerne l'aspect professionnel du rendu, la proximité avec le produit final et la facilité de tester le prototype (Ross, 2017). Cependant, ces outils sont dédiés à la conception de sites Web, donc surtout sur les objets d'interface classique (boutons, champs texte, etc.) et sur la navigation entre pages, tandis qu'un jeu vidéo est bien plus dynamique. On peut aussi envisager de s'appuyer sur un outil auteur de jeu en ligne simple d'utilisation - voire le même que celui utilisé pour le produit final - pour réaliser un jeu simplifié aisément manipulable et modifiable. Nous n'avons pas cédé à cette tentation, car il nous est apparu qu'on perdait un principe fort du prototypage papier, à savoir son immédiateté : changer une règle, ajouter un élément dans le jeu peut se faire quasiment immédiatement dans un prototype papier, même pendant le jeu, alors que, dans un outil auteur, il faut changer le programme, changer des paramètres ou bien éditer un sprite (image d'objet).

Une autre famille de solutions à laquelle nous avons songé est l'utilisation non plus d'un outil auteur, mais d'un environnement de jeu qui permettrait la communication dans un environnement virtuel lui-même physique et manipulable. Mais les jeux/environnements virtuels que nous avons pu analyser dans ce sens se sont avérés trop complexes à s'approprier, pour une manipulation limitée. Par exemple, le jeu Minecraft

\footnotetext{
${ }^{2}$ Une télémanipulation d'un prototype réel ne semble pas une solution réaliste.
} 
a été envisagé, mais il nous a semblé difficile de réaliser un prototype avec des éléments de toute forme que l'on contrôle dans un prototype papier de jeu, tout du moins avec notre propre expertise du jeu Minecraft. Finalement, nous nous sommes dirigés vers des outils 2D, à savoir tout simplement des tableaux blancs en ligne qui permettent de partager des dessins en temps réel. Trois critères sont apparus importants :

- I'utilisabilité, car il est essentiel que les gestes de manipulation des objets du prototype soient naturels, sans être entravés par des problèmes ergonomiques; dans ce critère, on inclura les latences éventuelles de l'outil;

- l'expressivité, à savoir la possibilité de disposer d'outils pratiques pour rapidement dessiner des objets; la possibilité d'importer ses propres objets ou images est indispensable, tandis que l'existence d'une bibliothèque interne d'objets disponibles constitue un plus;

- la conscience de l'autre (awareness), non pas en tant que simple participant à une réunion, mais comme co-manipulateur du même prototype.

Après avoir testé plusieurs outils, ce dernier critère a été discriminant et nous avons pu distinguer deux manifestations de celui-ci sous forme de caractéristique technique. La première caractéristique est la possibilité de visualiser en temps réel le pointeur de chacun des autres interacteurs. Ainsi, quand un participant dit « il faut agrandir cette zone " en voyant où le locuteur se trouve, il est important que l'interlocuteur comprenne ce à quoi il fait référence (problématique de gestion des déictiques que l'on retrouve dans les questions d'interactivité multimédia (Caelen, 1996)). La seconde caractéristique est plus subtile; elle concerne l'acte de manipulation lui-même : certains outils vont certes permettre des tracés et des déplacements en temps réel et fluides, mais les autres participants ne vont percevoir que le résultat du tracé ou du déplacement quand le manipulateur aura par exemple lâché sa souris après un glisserdéposer. À l'inverse, d'autres outils plus synchrones vont permettre de voir le tracé ou le déplacement pendant son exécution, ce qui peut sembler un détail s'il s'agit de créer collaborativement un schéma par exemple, mais qui est essentiel dans notre cas. En effet, un prototype papier sert à illustrer un processus, le processus de jeu au-delà de l'objet prototype lui-même. Un exemple très simple : quand, en phase de test, le joueur déplace un pion qui représente son personnage, il est essentiel de voir ce déplacement et non pas seulement le résultat du déplacement. Aussi, en phase de conception, voir les autres participants déplacer des objets permet de bien mieux comprendre ce qu'ils sont en train de faire, leur intention, un aspect essentiel dans la collaboration (Schwartz, 1998). Au final, nous avons choisi un logiciel en ligne appelé Idroo (Iteral Group OÜ, n.d.), un outil assez simple qui réunissait les critères ci-dessus (voir par exemple Guzairova, Bikmukhametov et Bakirova, 2019 pour un usage en apprentissage à distance). Pensé pour permettre à des enseignants de faire des démonstrations, notamment des démonstrations mathématiques, l'outil répondait particulièrement bien au critère d'awareness et de synchronicité. Par contre, il dispose de possibilités graphiques assez élémentaires et pas de bibliothèque d'objets tout prêts. D'autres concurrents, comme draw.io (diagrams.net, n.d.), apparaissaient beaucoup plus riches, avec un look plus moderne, mais n'étaient pas synchrones pour les déplacements.

Si Idroo prend en charge la médiatisation de la manipulation, qu'en est-il maintenant de la communication? Idroo inclut une messagerie et un clavardage audio, mais nous avons choisi de ne pas exploiter ces fonctionnalités, préférant utiliser des outils optimisés pour la communication. Sur ce plan, nous cherchions un service permettant de reproduire la structuration de la salle de cours telle qu'aménagée les années précédentes en îlots pour chacun des quatre à six jeux conçus par les étudiants. Beaucoup d'outils de visioconférence ne sont pas très pratiques quand il s'agit de circuler entre espaces de travail, ce qui constituait un besoin important: circulation de l'équipe enseignante dans les groupes, mais aussi circulation des étudiants qui testent mutuellement leurs prototypes ou bien assistent à une démonstration. 
Nous avons finalement retenu l'outil Zoom ${ }^{3}$, car il dispose d'une fonction permettant précisément de diviser les membres de la salle plénière en sous-salles. Deux autres outils de communication supplémentaires ont été adoptés. D'une part, Mattermost (Mattermost Inc., n.d.), une plateforme de messagerie instantanée déjà utilisée pour le cours, a eu deux fonctions : solution de secours en cas de perte de communication dans l'outil de visioconférence et possibilité d'avoir des échanges facilités entre salles via un canal de discussion commun. D'autre part, l'équipe enseignante s'est tournée vers Skype ${ }^{4}$ pour se coordonner. En effet, gérer toute la communication sur le même outil, Zoom, est difficilement envisageable, surtout avec ses sous-salles qui ne communiquent pas avec le reste des participants. Avec un outil séparé, on évite les confusions, l'outil nous indique à qui on communique. Ici, Skype a été choisi, car c'était le logiciel de communication usuel de l'équipe enseignante. Enfin, une simple page partagée, créée sous la forme d'un Google Document, a été rédigée afin d'expliquer le fonctionnement de l'ensemble et de constituer un « portail » recensant tous les outils. Cette architecture est représentée en figure 1.

${ }^{3}$ https://www.zoom.us/

${ }^{4}$ https://www.skype.com/ 
Figure 1

Cartographie des différentes applications de communication et de collaboration du dispositif

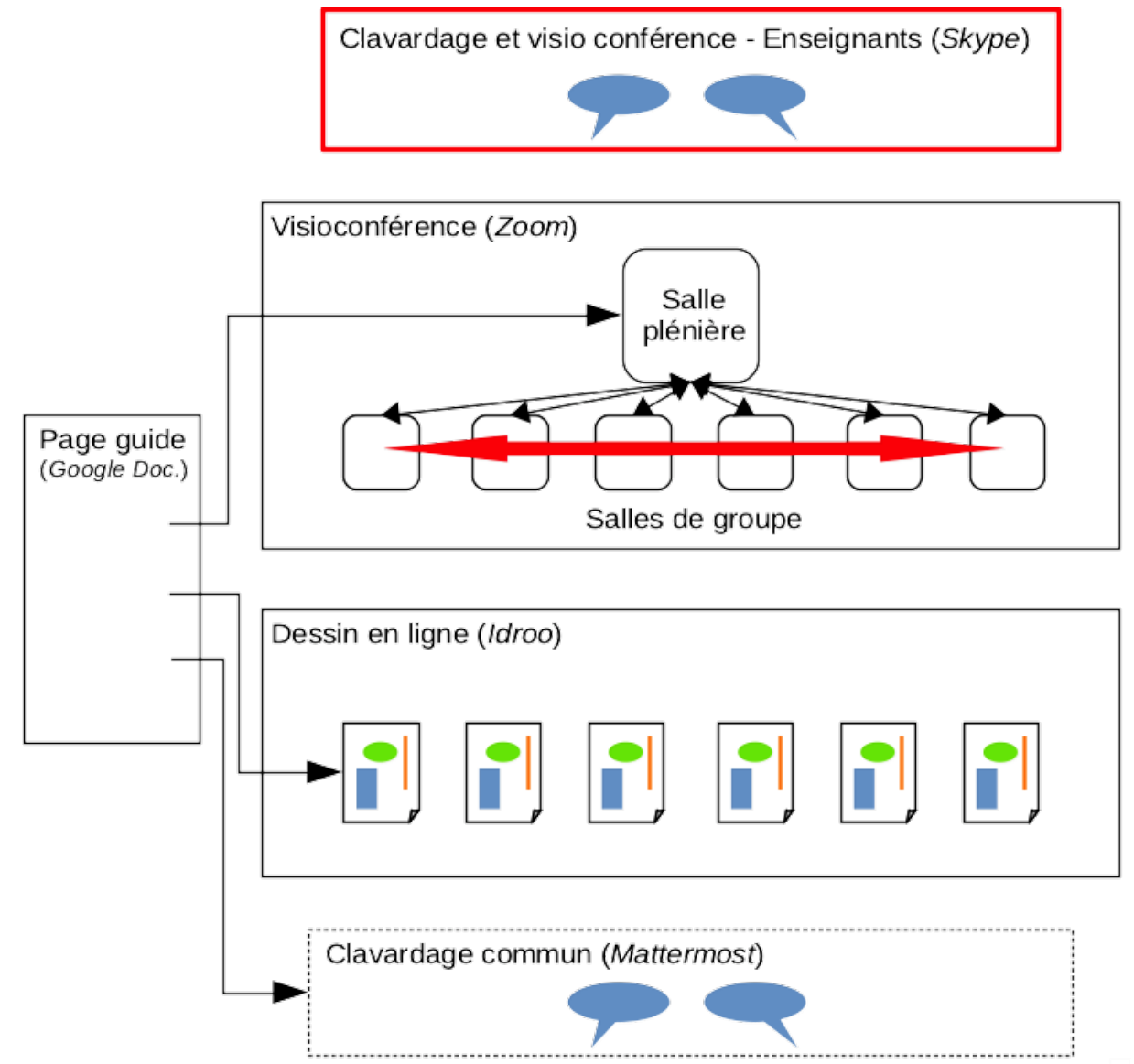

Note. Les flèches représentent les navigations possibles entre composants. Les parties en épais et rouge représentent les éléments spécifiques aux acteurs enseignants. Le clavardage commun est en pointillé pour signifier qu'il s'agit plus d'un outil de communication « de secours ».

\section{Déroulement de l'activité, perception de l'équipe enseignante}

Le cours sur les jeux vidéo pédagogiques s'est déroulé sur deux après-midi et deux journées entières. Lors de la première réunion Zoom, un tour de table virtuel a été d'abord mis en place afin de discuter brièvement de l'état d'avancement de chaque projet. Les apprenants ont ensuite été dispersés dans les sous-salles pour soulever les questions restées en suspens tandis que les enseignants naviguaient entre les différents groupes. La seconde après-midi de cette semaine de prototypage s'est ouverte avec la présentation et l'initiation au logiciel Idroo (créations des profils, démonstration des différentes actions exécutables, génération des tableaux blancs, etc.). L'activité de prototypage a été lancée dès lors et s'est poursuivie jusqu'à la fin de la semaine, entrecoupée par des phases de présentation de projets. La complexité du dispositif, sa nouveauté ainsi que les conditions matérielles et psychologiques propres à la crise sanitaire ont rendu la semaine particulièrement éprouvante tant pour l'équipe enseignante que pour les apprenants. En présentiel, l'activité est déjà intense, mais la mise à distance en urgence a demandé une prise en main rapide des outils et le jonglage entre les multiples logiciels prévus par le scénario. 
Sur le plan de la manipulation médiatisée, le logiciel Idroo a joué son rôle. Les apprenants se le sont approprié, la conception collaborative autour de l'artefact a pu avoir lieu. Cependant, avec des fonctionnalités d'édition plutôt basiques, illustrer ses idées dans le logiciel était parfois ardu. Nous avons pu constater, par rapport aux prototypes papier en présentiel, que le test d'un prototype était plus long, parfois près d'une heure, ce qui a diminué le nombre total d'itérations pour certains groupes.

Sur le plan de la communication médiatisée, l'étanchéité des salles s'est révélée être une limite pour les apprenants dans le sens où ils ne pouvaient pas voir ni essayer les jeux créés par les autres groupes et il n'y avait pas l'ambiance dynamique d'une salle de classe habituelle. Pour l'équipe enseignante, le fait de devoir se déplacer dans les différentes sous-salles s'est révélé laborieux, d'autant plus qu'il a fallu simuler le rôle de joueurs pour chaque test de prototypes, les élèves ne pouvant pas aisément circuler entre les salles. Le passage au format distanciel dichotomise les relations sociales : on est dans une salle ou on ne l'est pas. Tandis que, dans le réel, on est dans un groupe qui lui-même est à côté des autres groupes : on entend certaines paroles des voisins et l'effet cocktail party permet de réagir à certains propos même si l'attention est portée sur son propre groupe. Sur le plan visuel, on voit, de plus ou moins loin, les prototypes avancer et on peut s'en inspirer. Bref, en visioconférence, on perd totalement la dynamique de classe du format présentiel, même divisée en îlots, tout du moins dans le système que nous avons choisi ${ }^{5}$.

Malgré ces écueils, nous avons pu observer un certain nombre d'atouts de la distance, au-delà de la sécurité sanitaire, et des arguments classiques de la distance (allègement des salles de cours, diminution des transports). Nous avons pu faire intervenir des assistants externes « à la demande " pour fournir une aide ponctuelle sur une expertise particulière, un outil auteur par exemple. En présentiel, ce type d'intervention est beaucoup plus difficile à organiser. Dans le même ordre d'idées, certains prototypes de jeu ont pu être testés par une population extérieure au cours. Ainsi, Lina, neuf ans, a pu être la testeuse d'un jeu d'apprentissage sur les formes géométriques. Un autre atout de la distance a concerné le prototype lui-même, plus précisément pendant la phase de test du jeu. Les jeux rapides, par exemple les jeux de plateforme, ont toujours été difficiles à tester, car il faut faire bouger simultanément de nombreux éléments sur le prototype et le joueur tout comme les concepteurs ont du mal à s'y retrouver avec toutes les mains qui s'agitent sur le plateau de jeu. En mode virtuel, ces mains sont invisibles! Le joueur peut ainsi interagir avec un système dynamique, ne voyant pas chacun des membres du groupe de concepteurs déplacer tous les éléments en même temps. On a ainsi pu tester un jeu inspiré du jeu classique Lemmings avec une fluidité remarquable - non sans un effort important des étudiants « en coulisse ».

À la fin de la semaine, la grande satisfaction de l'équipe enseignante a été de constater que les prototypes virtuels avaient permis un avancement dans la conception similaire à ce que l'on avait pu observer avec les prototypes papier réalisés les années précédentes. Et en fin de compte, un mois plus tard, la qualité globale des jeux produits était très concluante, avec notamment deux jeux remarquablement aboutis ${ }^{6}$.

Cette vision plutôt positive de l'activité reste celle des enseignants et nous avons souhaité savoir si elle se reflétait chez les apprenants.

\footnotetext{
${ }^{5}$ Entre temps, nous avons découvert un nouvel outil de communication à distance, appelé theonline.town (Siempre Inc., n.d.), qui reproduit mieux la continuité spatiale des communications audio et visuelles.

${ }^{6}$ https://tecfa.unige.ch/jeux/jeux/\#617+category:2020
} 


\section{Perception des étudiants}

Afin d'avoir un retour sur la perception des étudiants relative à ce format de cours exceptionnel, nous leur avons soumis un questionnaire contenant cinq questions. Celles-ci sont inspirées des commentaires recueillis informellement lors de la semaine de cours et des perceptions des enseignants. Les deux premières questions visaient à vérifier l'hypothèse selon laquelle, malgré la re-scénarisation à la volée suboptimale, il n'y aurait pas aussi certains avantages dans le prototypage virtuel à distance. La troisième question avait pour but de mieux saisir les modalités de communication et de collaboration pendant l'activité. La quatrième question portait sur l'impression générale des étudiants tandis que la dernière recueillait des suggestions d'amélioration. Le questionnaire a été envoyé aux 19 étudiants; 11 ont répondu. Nous résumons ici leurs réponses.

Dans quelle(s) situation(s) avez-vous trouvé que le prototypage papier en présence aurait été préférable au prototypage à distance, et à quel(s) niveau(x)?

II ressort distinctement des avis recueillis que le prototypage papier est jugé plus adéquat pour cette semaine de développement, le format facilitant notamment le partage des idées grâce à la manipulation des matériaux physiques. Des remarques récurrentes renvoient à l'utilisation de Idroo, plus précisément au temps nécessaire pour se familiariser avec l'outil et à la difficulté à déplacer les objets virtuels, surtout pour les phases de test.

À l'inverse, dans quelle(s) situation(s) avez-vous trouvé que le prototypage à distance a offert des avantages que la même activité en présence n'aurait pu offrir? Pour quel(s) niveau $(x)$ ? Veuillez si possible détailler votre réponse.

Concernant les avantages, les utilisateurs ont valorisé les fonctions undo de Idroo, de même que la fonction d'ajout des multimédias externes. N'ayant pas à fabriquer manuellement le matériel des jeux, certains apprenants apprécient la manipulation de "vraies images », accélérant ainsi la conception de leurs prototypes, notamment lorsque le matériel des jeux devait être retouché. Un apprenant plébiscite le prototypage à distance, car ce format prédisposerait à un meilleur équilibre participationnel au sein du même groupe et éviterait ainsi l'effet de comparaison du niveau d'avancement avec les autres.

Nous pouvons déjà discuter les réponses à ces deux premières questions. De manière générale, la perception des apprenants est assez distincte de celle des enseignants exposée plus haut. En particulier, les apprenants focalisent leurs remarques sur l'outil de dessin Idroo, souvent en mettant en avant la qualité du résultat, soit positivement (possibilité d'importer ses images), soit négativement (en mentionnant d'autres outils jugés meilleurs, mais qui selon nous ne répondaient pas au cahier des charges, tels que Figma ou MarvelApp). Le point de vue des étudiants recoupe sur ce point un avantage identifié du prototypage virtuel par rapport au papier (Ross, 2017). Toutefois, les étudiants saisissent moins l'importance de l'immédiateté et de l'awareness dans l'outil choisi, cèdent parfois à la tentation du « joli prototype » qui rend bien, mais sert moins l'activité de conception elle-même : « When you are making a prototype, you do not need to be concerned with perfecting how it looks » (Fullerton, 2008). Notons qu'il s'agit là d'une tendance que nous avons pu aussi observer dans le prototypage papier.

\section{La modalité proposée vous a-t-elle permis de bien collaborer? Précisez dans quelle mesure et pourquoi.}

La plupart des apprenants mettent en exergue une bonne communication au sein de leur groupe, tout en affirmant que cela n'est pas nécessairement imputable à la modalité proposée. Certains étudiants disent être soulagés d'avoir pu remplacer le temps de trajets par des moments dévolus à l'avancement de leur projet. Par ailleurs, la pluralité des logiciels mobilisés a aussi largement plu aux apprenants, notamment 
le scénario prévu sur Zoom qui a comblé le manque de promiscuité physique. II semblerait que la synchronisation des dessins de Idroo, l'accès en tout temps au prototype malgré la distance spatiotemporelle ait aussi été une réelle plus-value pour les apprenants.

Pour des raisons assez diverses, parfois plus liée à la dimension pratique qu'aux composantes de l'ingénierie pédagogique, la collaboration a pu s'effectuer de manière satisfaisante. Nos données limitées ne nous permettent pas à ce stade d'identifier les éléments de la scénarisation pédagogique qui ont été décisifs.

\section{Globalement, comment avez-vous trouvé l'expérience?}

Sur onze personnes interrogées, cinq qualifient l'expérience de plutôt satisfaisante, trois sont mitigées, trois estiment qu'elle n'est pas très satisfaisante et une personne la considère comme très satisfaisante. On note que les avis sont partagés et un peu moins positifs que ceux de l'équipe enseignante. II convient de rappeler que ces perceptions globales de l'expérience pédagogique ont probablement été " contaminées » par le contexte anxiogène du cours : angoisse du confinement, difficultés matérielles et organisationnelles (certains de nos étudiants étaient pères ou mères de famille), retour de la peur de la pandémie dans nos sociétés (Villiot-Leclercq, 2020).

\section{Et si c'était à refaire?}

La situation exceptionnelle de mise en place de cette activité de prototypage nous a permis d'explorer des pistes d'ingénierie pédagogique que nous n'aurions jamais envisagées en temps normal. On retrouve ici une configuration connue : sous la contrainte, l'innovation et la créativité peuvent davantage s'exprimer. Globalement, la médiatisation du prototypage papier a fonctionné, comme en témoigne la qualité des prototypes et des jeux produits en fin de cours. Se pose alors la question : faut-il conserver cette modalité pour les itérations à venir du cours? Cette même question s'est d'ailleurs posée concernant le télétravail en général au sortir de la période de confinement dans laquelle s'est déroulée l'activité décrite.

Cette question est d'autant plus pertinente que certains couacs du cours peuvent facilement être évités si on scénarise le cours dans son ensemble, et non en urgence et à la volée. Ainsi, les apprenants se sont plaints de ne pas avoir été formés au préalable aux outils (notamment Idroo). Dans le contexte d'urgence, nous avons renoncé à introduire l'outil avant la semaine de prototypage distancielle, d'une part par manque de temps, mais aussi pour ne pas encore surcharger les apprenants qui eux aussi vivaient une période de stress important. Dans une scénarisation normale, une formation préalable peut être organisée, et c'est le cas pour d'autres outils du cours.

Notre position est que la mise à distance de cette activité tangible pourrait tout à fait s'effectuer dans la durée, à condition de repenser quelque peu le scénario pour mieux préparer et accompagner dans l'activité, et globalement pour la rendre plus fluide, moins stressante. Mais le choix entre présence et distance, entre matérialité du prototype tangible et virtualité des tableaux blancs partagés, entre dynamique naturelle de classe et régulation électronique des communications est un choix de valeur. Le confinement du printemps 2020 nous a montré la possibilité d'une totale virtualité des activités intellectuelles, mais aussi l'effrayante dystopie qui accompagne cette possibilité, à savoir un monde où les êtres humains ne sont connectés que par les réseaux. Dans une formation dédiée aux technologies numériques, le prototypage papier doit rester un îlot de déconnexion, une bouffée d'oxygène pour nous rappeler que la créativité collective passe préférentiellement par la matière et le corps. 


\section{Liste de références}

Axure. (n.d.). Axure [logiciel]. https://www.axure.com/

Caelen, J. (1996). Interaction et multimodalité. Dans Troisième colloque Hypermédias et Apprentissages (p. 11-32). Récupéré le 30 août 2020 de https://edutice.archives-ouvertes.fr/edutice-00000506

Charlier, B., Deschryver, N. et Peraya, D. (2006). Apprendre en présence et à distance. Une définition des dispositifs hybrides. Distances et Savoirs, 4(4), 469-496.

Conrad, D. (2005). Building and maintaining community in cohort-based online learning. International Journal of E-Learning \& Distance Education/La revue Internationale de l'apprentissage en ligne et de l'enseignement à distance, 20(1), $1-20$.

diagrams.net. (n.d.). draw.io [logiciel]. https://www.draw.io/

Figma. (n.d.). Figma [logiciel]. https://www.figma.com/

Fullerton, T. (2008). Game Design Workshop: A Playcentric Approach to Creating Innovative Games. Amsterdam: Morgan Kaufmann.

Guzairova, G. R., Bikmukhametov, I. K. et Bakirova, R. R. (2019). The use of virtual distance learning technologies in conducting pre-study courses in mathematics to prepare for the unified state examination at a specialized level. Dans Proceedings of $1^{\text {st }}$ International Scientific and Practical Conference on Digital Economy (ISCDE 2019) (p. 612-618). Récupéré le 30 août 2020 de https://doi.org/10.2991/iscde-19.2019.118

Iteral Group OÜ. (n.d.). IDroo [logiciel]. https://idroo.com/

Marvel. (n.d.). Marvel [logiciel]. https://marvelapp.com/

Mattermost Inc. (n.d.). Mattermost [logiciel]. https://mattermost.com/

Peraya, D. (2008). Un regard critique sur les concepts de médiatisation et médiation: nouvelles pratiques, nouvelle modélisation. Les Enjeux de l'information et de la communication, 9(2). https://lesenjeux.univ-grenoblealpes.fr/2008/supplement-a/12-un-regard-critique-sur-les-concepts-de-mediatisation-et-mediation-nouvellespratiques-nouvelle-modelisation

Ross, J. (2017). Prototyping: Paper Versus Digital. Récupéré le 30 août 2020 de https://www.uxmatters.com/mt/archives/2017/05/prototyping-paper-versus-digital.php

Scanlon, E., Colwell, C., Cooper, M. et Di Paolo, T. (2004). Remote experiments, re-versioning and re-thinking science learning. Computers \& Education, 43(1), 153-163. https://doi.org/10.1016/j.compedu.2003.12.010

Schell, J. (2008). The Art of Game Design. CRC press.

Schwartz, D. L. (1998). The productive agency that drives collaborative learning. Dans P. Dillenbourg (dir.), Collaborative learning: Cognitive and computational approaches (p. 197-218). New York: Elsevier.

Siempre Inc. (n.d.). Online Town [logiciel]. https://theonline.town/

Villiot-Leclercq, E. (2020). L'ingénierie pédagogique au temps de la Covid-19. Distances et Médiations Des Savoirs, 30. Récupéré le 30 août 2020 de http://journals.openedition.org/dms/5203 